\title{
Establishing a perioperative medicine for older people undergoing surgery service for general surgical patients at a district general hospital
}

\author{
Authors: Ruth de Las Casas, ${ }^{A}$ Catherine Meilak, ${ }^{B}$ Anna Whittle, ${ }^{B}$ Judith Partridge, ${ }^{C}$ Jacek Adamek, ${ }^{D}$ Euan Sadler, ${ }^{E}$ \\ Nick Sevdalis ${ }^{F}$ and Jugdeep Dhesi ${ }^{G}$
}

\section{Introduction}

There is growing recognition of the need for perioperative medicine services for older surgical patients. Comprehensive geriatric assessment and optimisation methodology has been successfully used to improve perioperative outcomes at tertiary centres. This paper describes translation of an established model of geriatrician-led perioperative care to a district general hospital (DGH) setting.

\section{Methods}

A mixed methods quality improvement programme was used and included stakeholder co-design, identification of core components, definition of mechanisms for change, and measurement of impact through qualitative and quantitative approaches.

Results

Within 18 months, a substantive perioperative service for older people was established at a DGH, funded by the surgical directorate. Key outcomes included reduction in length of stay and 30-day readmission and positive staff and patient experience.

\section{Discussion}

This study is in keeping with improvement science literature demonstrating the importance of a mixed-methods approach in translating an evidenced-based intervention into another setting, maintaining fidelity and replicating results.

Authors: ${ }^{A}$ anaesthetic trainee and darzi fellow, Guy's and St Thomas' NHS Foundation Trust, London, UK; ${ }^{\text {B }}$ consultant geriatrician, Dartford and Gravesham NHS Trust, Kent, UK: 'consultant geriatrician, Guy's and St Thomas' NHS Foundation Trust, London and honorary senior lecturer, King's College London,

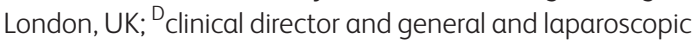
colorectal consultant surgeon, Dartford and Gravesham NHS

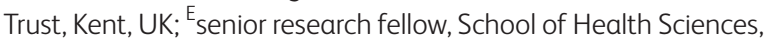
University of Southampton, Southampton, UK; ${ }^{\text {F }}$ professor of implementation science \& patient safety, Centre for Implementation

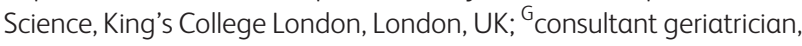
Guy's and St Thomas' NHS Foundation Trust, London, UK, honorary reader, King's College London, London, UK, and honorary associate professor, University College London, London, UK
KEYWORDS: perioperative medicine for older people undergoing surgery, POPS, comprehensive geriatric assessment, quality improvement, implementation science, frailty, surgery

DOI: 10.7861/clinmed.2021-0356

\section{Introduction}

Patients aged over 60 years are more likely than younger people to develop complications following surgery. ${ }^{1}$ With each additional decade of life, postoperative morbidity, mortality and length of in-hospital stay (LOS) increase. ${ }^{2}$ Comanagement between surgeons, anaesthetists and geriatricians is increasingly recognised to improve postoperative outcomes. ${ }^{3}$ Collaborative perioperative services using comprehensive geriatric assessment (CGA) methodology have demonstrated fewer postoperative complications in patients undergoing orthopaedic, ${ }^{4}$ vascular ${ }^{5}$ and general surgical procedures. ${ }^{6}$ These observed improvements with consequent shorter LOS may provide a cost-effective approach to delivering services for older surgical patients.

The UK, Royal Colleges of Anaesthetists, Surgeons and Physicians all advocate collaborative models of perioperative care. $^{7-9}$ Indeed, the Royal College of Surgeons report The high risk general surgical patient and the National Emergency Laparotomy Audit (NELA) recommend patients aged 65 years or over should be assessed by a geriatrician during a hospital episode. ${ }^{10,11}$ Despite these standards, implementation of geriatric medicine services in routine surgical care (excepting hip fracture) remains limited. NELA data show only $23 \%$ of older patients undergoing emergency laparotomy are reviewed by a geriatrician, and structured geriatrician-surgical liaison exists in just $4 \%$ of hospitals. ${ }^{11}$

Barriers to establishing perioperative geriatrician involvement include insufficient funding and a limited workforce to deliver collaborative comanagement. ${ }^{12}$ Furthermore, qualitative research describes human factors and departmental cultures presenting barriers to change. ${ }^{13}$ Despite these barriers, national drivers, education and training opportunities and coproduced medical-surgical business plans have resulted in an increase in geriatrician-delivered perioperative medicine services. ${ }^{14,15}$ While this is encouraging, there remains a translation gap between 
recommendations and clinical practice. Achieving systematic scale-up requires an implementation science approach to define and address barriers and enablers, ensure fidelity to evidence and facilitate measurement of outcome.

This mixed methods study aims to address this implementation and scale-up gap through effectively and sustainably translating an established model of geriatrician-led perioperative care from the tertiary centre where it was established, to a district general hospital (DGH).

\section{Methods}

\section{The intervention:POPS@GSTFT}

The 'Perioperative medicine for Older People undergoing Surgery' (POPS) service was established at Guy's and St Thomas' Foundation Trust (GSTFT) in 2003. GSTFT is a 1,277 bedded tertiary, academic hospital with 28,000 elective and 51,500 nonelective admissions annually. Ninety-one percent of emergency general surgical patients were seen by a geriatrician, ${ }^{2}$ with LOS shorter than the national benchmark in 2016. POPS uses CGA methodology, delivered by a geriatrician-led multidisciplinary team to preoperatively assess and optimise in a one-stop clinic with a focus on anticipation of postoperative complications and shared decision making. Patients are followed up on surgical units with comanagement between geriatricians and surgeons to deliver postoperative medical care, rehabilitation goal setting and discharge planning. Emergency admissions are identified through proactive case finding and managed (pre- and postoperatively) according to CGA principles. The POPS model of care is illustrated in supplementary material S1. Single-site studies have demonstrated improvements in clinical outcomes, process measures and staff satisfaction. ${ }^{4,5}$

\section{The context for scale-up: Dartford and Gravesham Trust}

Darent Valley Hospital (Dartford and Gravesham Trust, DGT) is a 463 bedded hospital, 16 miles from GSTFT serving a population of 340,000 with a deprivation index of $100 / 152{ }^{16}$ Trustwide issues include workforce shortages, financial challenges and an anticipated increase in activity. ${ }^{17}$ The surgical department has 2,000-3,000 emergency and 1,100-1,500 elective admissions annually. Performance, measured by NELA, showed $8 \%$ of emergency general surgical patients were seen by a geriatrician in 2016, and LOS was longer than the national benchmark. ${ }^{2}$

Funding from the NHS England Acute Care Collaboration Vanguard provided the opportunity to translate POPS@GSTFT to the DGT setting. Over 18 months, $£ 25,000$ was provided to support one weekly session of POPS@GSTFT consultant time supervising 2 days of specialist registrar ( $S p R)$ time to implement the POPS@DGT service.

\section{The translation process: POPS@DGT}

Improvement science strategies were employed to facilitate the translation of POPS@GSTFT to DGT. ${ }^{18,19}$ The three main phases were:

\section{Logic modelling}

The core components of POPS@GSTFT were described through development of a modified logic model. ${ }^{20}$ Logic models specify elements of a clinical programme, and how they translate to care processes and resultant clinical outcomes. They also specify the core elements of a clinical intervention to be delivered with high fidelity, ${ }^{21}$ ensuring the maintenance of effectiveness through the scale-up process. In so doing, logic models offer a practical, actionorientated 'map' for the execution of a translation or scale-up programme. 22

\section{Piloting the service}

Using the logic model, a pilot POPS@DGT service was established. The first phase of this pilot service provided outpatient, preoperative CGA-based, geriatrician-delivered care for older patients scheduled to undergo vascular surgery. This early service comprised:

> Staffing: 2 days per week of geriatric medicine SpR with expertise in delivering CGA, and a weekly session of supporting POPS@GSTFT consultant time (eg review of clinic letters, supervision delivered through weekly multidisciplinary meetings).

> Supporting clinical infrastructure:POPS@GSTFT written materials (eg clinic proformas, letter templates, clinical guidelines, Standard Operating Policies).

> Professional development: mentoring of registrars by POPS@ GSTFT team to build resilience and sustainability.

Piloting a small-scale service in one surgical speciality before expanding across other surgical specialities facilitated interdisciplinary relationships, improved understanding of local processes, and allowed anticipation of enablers and barriers to wider implementation. Furthermore, it provided the opportunity for 'troubleshooting', while allowing the local team to 'own' and 'invest' in the intervention. ${ }^{17}$

\section{Iterative evaluation to support embedding of POPS@ DGT}

POPS@DGT was studied using a clear-box, formative service evaluation. This allowed granular description of how inputs translated into outputs, and provided real-time insight into modification, refinement and tailoring of the intervention. ${ }^{23,24}$

Quality improvement methodology enabled continual adaptation of the intervention to the local context. Process mapping with local stakeholder groups provided understanding of howPOPS@DGT could be embedded into existing systems. Where areas for improvement were identified, Cause and Effect Fishbone diagrams, the Five Whys approach ${ }^{25}$ and Plan-Do-Study-Act cycles ${ }^{26}$ were used to analyse root cause of challenges, identify solutions and trial modifications in a stepwise manner.

Staff feedback, via semistructured interviews and surveys, was central to service development. For example, the format and content of ward-based multidisciplinary meetings (MDMs) was refined through PDSA cycles, allowing POPS@DGT to make iterative adjustments to time, venue, and frequency of meetings, until staff agreed the MDM was an efficient use of time and improved clinical care.

Improvement steps to facilitate translation, together with the timeline for change, are summarised in Table 1.

Through phases 1 to 3,POPS@DGT expanded to include inpatient pre- and postoperative care for emergency and elective general surgical patients. Capacity for expansion was achieved through a funding application using preliminary data resulting in 
Table 1. Summary of evaluation KPIs and associated improvement steps over time for POPS@DGT scale-up Timeline Oct 2017 - Sept 2018

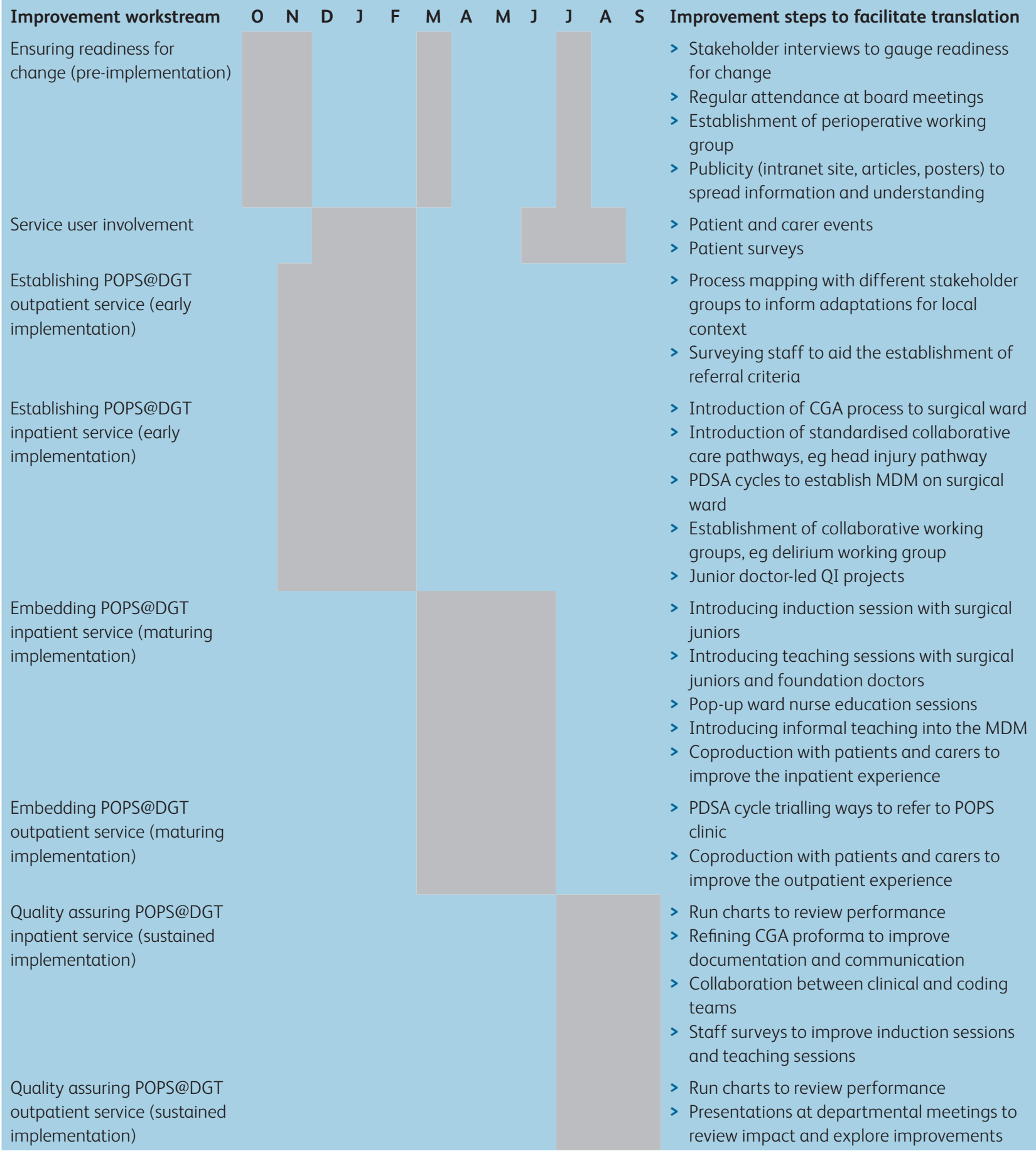

the appointment of a surgical directorate funded clinical nurse specialist (CNS).

\section{POPS@DGT: evaluation of translation}

In keeping with an implementation science approach, a mixed methods evaluation of the POPS@DGT intervention was used. A hybrid evaluation framework assessing process (clinical and implementation), stakeholder, and patient satisfaction data was used $^{27}$ to achieve sustainability.

Baseline data were collected on a cohort of 50 emergency and 50 elective general surgical patients aged 70 years or over. Following introduction of POPS@DGT, quantitative data on all patients seen by POPS were presented in monthly run charts recording LOS, readmissions, medical complications and coding of comorbidities. 
Qualitative data were obtained using semistructured interviews and stakeholder focus groups, analysed using NVIVO 12 software.

\section{Results}

Results are presented in three sections. First, quantitative data allow evaluation of the clinical impact of POPS@DGT (similar to original evidence from GSTFT). Second, qualitative data describe challenges experienced through implementation and allow exploration of barriers, enablers and coproduction of interventions to overcome these challenges. Third, the process of obtaining substantive funding and the current POPS@DGT service is described.

\section{Process measures, staff-reported and patient- reported outcomes}

Uptake (implementation assessment)

In the 12 months preceding POPS intervention, $8 \%$ of patients undergoing emergency laparotomy were seen by a geriatrician. During the first 12 months of the POPS intervention, $98 \%$ of patients aged over 70 years undergoing emergency laparotomy received CGA ( $n=62)$ with one early postoperative death in critical care precluding intervention. Within their first 12-month period, POPS@DGT performed a CGA on a total of 763 inpatients. Initially, CGA and optimisation were undertaken by POPS physicians (geriatric medicine registrars), but capacity was increased through upskilling a CNS to undertake CGA and optimisation.

\section{Clinical outcomes}

The incidence of documented postoperative complications increased over the first year of POPS@DGT: delirium from 0\% to $26 \%$, acute kidney injury $2 \%$ to $14 \%$ and pneumonia $10 \%$ to $19 \%$ (Fig 1). These increases likely reflect improved recognition of complications, rather than an increase in complication rates. Comorbidities recorded on discharge summaries increased from a median of two to four per patient. Again, this likely reflects improved recognition and documentation rather than increased multimorbidity, and was achieved through measures such as an 'Information to include in the Discharge Note' section in the POPS@DGTCGA proforma.

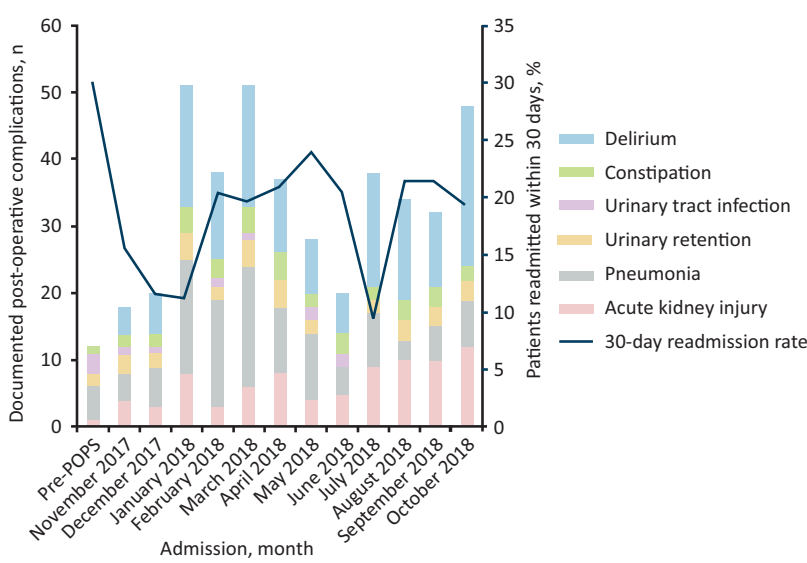

Fig 1. Postoperative medical complications and 30-day readmission rate in emergency general surgical patients.
Within 12 months of introduction of POPS@DGT, median LOS for emergency general surgery patients reduced by 1 day and mean LOS by 2.58 days (pre-POPS: median 8 , mean 14.2 , range $2-80$ days; post-POPS: median 7 , mean 11.6 , range $1-82$ days). Within 12 months of introduction of POPS@DGT 30-day readmissions in emergency general surgical patients reduced from 30-18\% (Fig 1).

\section{Staff-reported outcomes}

Nine months after service introduction an electronic survey distributed to nurses, doctors and allied health professionals found $>80 \%$ of staff reported POPS@DGT had improved the overall care of older surgical patients ( $n=28$, response rate $71 \%$ ). In addition, staff reported improved understanding of multidisciplinary working and enhanced educational opportunities. Interviews with stakeholders demonstrated improved satisfaction with discharge letter documentation, noted particularly by general practitioners.

\section{Patient-reported outcomes}

Patients attending POPS clinics were invited to attend a focus group supported by an independent facilitator. Patients who expressed interest but were unable to attend were invited to complete a survey (supplementary material S2). This process enabled iterative codesign of the service by rapidly identifying aspects requiring adaptation and facilitating coproduced solutions. Improvements made included changes to the appointment process (specifically flexibility in appointment times and content of letters), a coproduced information leaflet (supplementary material S3), and the development of a hospital map. The success of this approach was recognised through the substantive appointment of a patient engagement officer working across DGT, demonstrating sustainable cultural change within the organization.

\section{Qualitative evaluation of the translation process}

Semistructured interviews were conducted 6 months after service introduction with 10 senior staff members involved in POPS implementation at DGT. Staff interviewed were two nurses, two surgeons, three geriatricians, two anaesthetists and one member of the executive hospital board. Interviews explored individuals' experience of the implementation process: had there been readiness for change, had sufficient stakeholder engagement been achieved, had the intervention been perceived as acceptable and had it adapted sufficiently into its new context? Thematic analysis of these transcribed interviews using NVIVO 12 software allowed an understanding of barriers and enablers to translation and interventions necessary to overcome challenges (Table 2).

\section{Sustainability}

Following the pilot, a business case was submitted and the substantive POPS@DGT service started in November 2017. This service, funded through the surgical directorate, is now provided by 1.0 working time equivalent (WTE) consultant geriatricians, 1.0 WTE band 7 CNS, 0.3 WTE occupational therapist and 0.1 WTEPOPS@GSTFT consultant. The inpatient service is delivered through daily POPS consultant and CNS comanagement on general surgical wards, close collaborative working with surgical teams, twice-weekly ward-based MDMs, and referral to community services at hospital discharge. A preoperative CGA-based and optimisation-based outpatient service has now been established. One session of POPS@GSTFT consultant time continues to provide clinical supervision, collaborative peer support and mentorship. 
Table 2. Implementation process of POPS@DGT: barriers and enablers

\begin{tabular}{|c|c|}
\hline Challenge & Enablers \\
\hline $\begin{array}{l}\text { Achieving } \\
\text { organisational } \\
\text { readiness for } \\
\text { change }\end{array}$ & $\begin{array}{l}\text { > NHS England money available to pump-prime project funding } \\
\text { > Resources, ie staffing in place } \\
>\text { Clinical and implementation expertise on-hand from GSTFT } \\
>\text { Chief executive with belief in benefit of service: 'the right } \\
\text { thing to do' } \\
>\text { Success in National awards, ie shortlisting for BM] and HS] } \\
\text { awards, raising trust profile }\end{array}$ \\
\hline $\begin{array}{l}\text { Achieving } \\
\text { individuals' } \\
\text { readiness for } \\
\text { change }\end{array}$ & $\begin{array}{l}\text { Some staff perceived current service as not adequately } \\
\text { addressing needs of older patients, generating desire/tension } \\
\text { for change. More pronounced among nurses and AHPs } \\
\text { > Sessions for clinical and management staff demonstrated } \\
\text { purpose of service and potential improvements, setting a } \\
\text { shared vision } \\
\text { > Proactive engagement of staff throughout implementation } \\
\text { process (through conception, introduction, and improvement } \\
\text { work) facilitated 'buy-in' } \\
\text { Individuals with prior experience of working with similar } \\
\text { models of care in other centres were early acceptors and } \\
\text { advocates of service }\end{array}$ \\
\hline
\end{tabular}

Ensuring acceptability of intervention

Achieving multi-
disciplinary working over silo working

\section{Barriers}

$>$ Negativity regarding the likelihood of investment in new projects resulted in weak belief among clinical staff in their ability to deliver change, and therefore poor collective commitment to change

> Some staff perceived current service as working adequately, generating limited desire for change. More pronounced among doctors. Example comment 'We managed before you'

> Some individuals described introducing change as synonymous with introducing more work, leading to reluctance to engage

> Service implementation encountered individuals resistant to change throughout. The reasons could not always be understood or defined. Commitment to consistently delivering quality clinical work proved more powerful than other methods of persuasion for this group

> Evidence for the service (both published and anecdotal) arose from tertiary centre. Some perceived this as irrelevant due to different context of DGH

$>$ Achieving buy-in from junior surgical doctors was challenging with a 'surgery vs medicine' culture. Initially 'medical' jobs were perceived as lower priority than 'surgical' jobs

> Different working patterns hindered streamlined communication between teams, ie different handover times / consultant ward round times.

> Challenging traditional role definition, - ie geriatricians offering preoperative assessment provoked mixed opinions, especially from anaesthetists

$\mathrm{AHP}=$ allied health professional; BM] = British Medical Journal; $\mathrm{HS}]=$ Health Services Journal

Effective knowledge mobilisation through adaptation of POPS@GSTFT resources to the local context of POPS@DGT improved efficiency in the set-up phase and ensured consistency in documentation and approach across the vanguard sites. For example with respect to delirium; a pan-hospital delirium working group was established, a high-visibility sticker for use in the medical record was adapted, pop-up training sessions for nurses were provided using GSTFT training materials and dissemination occurred via newsletter articles and an in-hospital promotional event. In addition, the preoperative outpatient service utilises the same CGA tools and letter template as that used by POPS@GSTFT, facilitating seamless transfer of patient-level information between centres.

\section{Discussion}

Key findings

Through a mixed methods study, underpinned by improvement and implementation science, the establishedPOPS@GSTFT service was sustainably translated to and substantively funded at a DGH. Within 12 months, POPS@DGT demonstrated a 1-day reduction in median LOS and a reduction in 30 -day readmission rate $(30 \%$ to $18 \%)$ in older surgical patients. Detection of medical complications and comorbidity coding improved. Staff and patient benefits were demonstrated through mixed methods evaluation. 


\section{Interpretation}

Successful and sustainable translation of an evidence-based approach to a novel setting requires fidelity to the original model (core intervention elements), adaption to the local context (adaptable elements) and the use of improvement science to underpin iterative change.

Ensuring fidelity to POPS@GSTFT necessitated description and adherence to the core components of the intervention. ${ }^{21}$ The use of CGA and optimisation as an underpinning methodology is essential, as evidenced by previous work, with benefits observed in process and clinician-reported measures. ${ }^{4-6}$ In contrast, when principles of CGA are not adhered to, the same results are not observed. For example, in a colorectal cancer population, CGA failed to use a timely or multidisciplinary approach and comparable postoperative benefits were not demonstrated. ${ }^{28}$ Similarly, a recent study examining a 'toolkit' approach to perioperative CGA delivery by non-geriatricians, failed to develop and embed a clinical service and showed no impact on clinical outcomes. $^{29}$

While core components of CGA are integral to a POPS intervention, the process of translation requires adaption to the local context. In the POPS@DGT programme, local adaption was facilitated through a 'one team' approach supported through collaborative training, upskilling all staff in perioperative geriatric medicine. Codesign and coproduction involving stakeholders early in the process were effective in promoting shared service ownership and instrumental in securing substantive funding from the surgical directorate within 15 months of inception. This move towards funding POPS services from surgical as opposed to medical budgets is increasingly observed in the UK and likely further reflects shared understanding of a 'one team' approach. ${ }^{11}$

Finally, using a systematic approach to address human factors, known to be barriers to establishing team-based services, was essential. This is pertinent as a recent qualitative study suggests failure to address human factors may contribute to slow uptake of geriatrician-led perioperative medicine services. ${ }^{13}$ Furthermore funded clinical support and mentorship from the established POPS@ GSTFT team fostered resilience in POPS@DGT staff. This suggests the need for a national or international POPS network to share best practice, promote resilience and ensure sustainability at scale. ${ }^{11}$

\section{Limitations}

First a single-site translation study lacks the benefit of a control group making it impossible to infer causal attribution of the clinical and implementation outcomes observed. However, undertaking a pilot site translation, such as this study, is an integral first step in wider scale-up. Second, an unblinded evaluation team was a potential source of bias. Such limitations reflect the pragmatic choice of a formative evaluation, aimed at maximising learning from the implementation process. This necessitates a multicentre hybrid effectiveness-implementation trial to build on this translation study and evaluate wider scale-up of the POPS intervention. $^{27}$

\section{Conclusion}

There is a national and international appetite for establishing geriatrician-led perioperative medicine services. This study applied implementation and improvement science methods to successfully and sustainably translate an evidence-based approach from a tertiary setting to a DGH. Key components included use of a logic model, opportunistic pilot funding, ensuring fidelity to evidence, adapting to the local context and addressing human factors in coproducing services. Such approaches should be used in the wider scale-up and evaluation of POPS services in the future.

\section{Summary}

\section{What is known?}

Postoperative outcomes worsen with increasing age. Services using comprehensive geriatric assessment (CGA) methodology throughout the perioperative pathway demonstrate improvement in postoperative outcomes. Despite clear standards of care recommending assessment by a geriatrician in high-risk surgical groups, implementation of geriatric medicine services in routine surgical care (other than hip fracture) remains limited.

\section{What is the question?}

Is it possible to effectively and sustainably translate an established model of geriatrician-led perioperative care (Perioperative medicine for Older People undergoing Surgery (POPS)) from a tertiary centre to a district general hospital setting?

\section{What was found?}

A sustainable and substantively funded POPS service was established at a district general hospital. Within 18 months the service demonstrated reduction in length of stay and readmission rate, and improvement in patient- and staff-related outcomes.

\section{What is the implication for practice now?}

Quality improvement methodology should be used to facilitate the wider systematic scale-up of sustainable POPS services.

\section{Supplementary material}

Additional supplementary material may be found in the online version of this article at www.rcpjournals.org/clinmedicine:

S1 - POPS model of care graphic

S2 - POPS patient survey

S3 - POPS co-produced information leaflet

\section{Acknowledgements}

The authors would like to thank Michael Bedford for his assistance in database development and statistical analysis, and Donna Wallace for her expertise in project management.

\section{Conflicts of interest}

N Sevdalis is the director of London Safety and Training Solutions Ltd, which undertakes patient safety and quality improvement advisory and training services for healthcare organisations internationally.

\section{References}

1 Polanczyk CA, Marcantonio E, Goldman L et al. Impact of age on perioperative complications and length of stay in patients under-going non-cardiac surgery. Ann Intern Med 2001;134:637-43. 
2 NELA Project Team. Third patient report of The National Emergency Laparotomy Audit. Royal College of Anaesthetists London, 2017. www.nela.org.uk/Third-Patient-Audit-Report\#pt [Accessed 1 June 2021].

3 Ellis G, Sevadalis N. Understanding and improving multidisciplinary team working in geriatric medicine. Age Ageing 2019;48:498-505.

4 Harari D, Hopper A, Dhesi ] et al. Proactive care of older people undergoing surgery ('POPS'): designing, embedding, evaluating and funding a comprehensive geriatric assessment service for older elective surgical patients. Age Ageing 2007;36:190-6.

5 Partridge JS, Harari D, Martin FC et al. Randomized clinical trial of comprehensive geriatric assessment and optimization in vascular surgery. Br J Surg 2017;104:679-87.

6 Shipway D, Koizia L, Winterkorn $\mathrm{N}$ et al. Embedded geriatric surgical liaison is associated with reduced inpatient length of stay in older patients admitted for gastrointestinal surgery. Future Healthcare ] 2018:5:108-16.

7 Royal College of Anaesthetists. Perioperative medicine - the pathway to better surgical care. The Royal College of Anaesthetists London, 2015. www.rcoa.ac.uk/sites/default/files/PERIOP-2014.pdf [Accessed 1 June 2021].

8 Pearce L, Bunni J, McCarthy K, Hewitt J. Surgery in the older person: training needs for the provision of multidisciplinary care. Ann R Coll Surg Engl 2016;98:367-370.

9 RCP. Future hospital: caring for medical patients. The Royal College of Physicians, London 2013. www.rcplondon.ac.uk/projects/outputs/ future-hospital-commission [Accessed 1 June 2021].

10 Royal College of Surgeons. The high risk general surgical patient: raising the standard. RCS, 2018. www.rcseng.ac.uk/-/media/files/ rcs/library-and-publications/non-journal-publications/higher_risk_ surgical_patient_2011_web.pdf [Accessed 1 JUne 2021].

11 NELA Project Team. Fourth patient report of the National Emergency Laparotomy Audit. Royal College of Anaesthetists London, 2018. www.nela.org.uk/Fourth-Patient-Audit-Report\#pt [Accessed 1 June 2021].

12 Partridge J, Collingridge G, Gordon A et al. Where are we in perioperative medicine for older surgical patients? A UK survey of geriatric medicine delivered services in surgery. Age Ageing 2014;43:721-4.

13 Howie S, Tinker A. Are we on the same page? Exploring the role of the geriatrician in the care of the older surgical patient from the perspective of surgeons and geriatricians. Clin Med (Lond) 2018;18:374-9.

14 Joughin A, Partridge J, O'Halloran T, Dhesi J. Where are we now in perioperative medicine? Results from a repeated UK survey of geriatric medicine delivered services for older people. Age Ageing 2019;48:458-62

15 Thillainadesan J, Hilmer S, Close J, Kearney L, Naganathan V. Geriatric medicine services for older surgical patients in acute hospitals: results from a binational survey. Australas ] Ageing 2019;38:278-83.

16 Kent County Council. The English Index of Deprivation (IMD 2015): headline findings for Kent. Business Intelligence Statistical Bulletin October 2015. Strategic Business Development and Intelligence, Kent County Council. www.kent.gov.uk/__data/assets/
pdf_file/0006/7953/Indices-of-Deprivation-headline-findings.pdf [Accessed 1 June 2021].

17 Foundations for change: The development of the Foundation Healthcare Group 2016/17. Dartford and Gravesham Trust, Guy's and St Thomas' NHS Foundation Trust, 2017. www.guysandstthomas. nhs.uk/resources/innovation/Vanguard-End-of-Year-Report.pdf [Accessed 1 June 2021].

18 Powell B], Waltz T], Chinman M] et al. A refined compilation of implementation strategies: results from the Expert Recommendations for Implementing Change (ERIC) project. Implement Sci 2015;10:21.

19 Rogal SS, Yakovchenko V, Waltz T] et al. The association between implementation strategy use and the uptake of hepatitis $\mathrm{C}$ treatment in a national sample. Implement Sci 2017;12:60.

20 Jasper EV, Dhesi JK, Partridge JS, Sevdalis N. Scaling up perioperative medicine for older people undergoing surgery (POPS) services; use of a logic model approach. Clin Med (Lond). 2019;19:478-84.

21 Carroll C, Patterson M, Wood S et al. A conceptual framework for implementation fidelity. Implement Sci 2007:2:40.

22 Rogers PJ. Using programme theory to evaluate complicated and complex aspects of interventions. Evaluation 2008;14:29-48.

23 Scriven M. Three boxes: the fine line between evaluation and explanation. Evaluation Practice 1994;15:75-7.

24 The Health Foundation. Evaluation, what to consider: commonly asked questions about how to approach evaluation of quality improvement in health care, 2015. www.health.org.uk/publications/ evaluation-what-to-consider [Accessed 1 June 2021].

25 NHS Institute for innovation and improvement. The handbook of quality and service improvement tools. NHSI, 2010. webarchive. nationalarchives.gov.uk/20160805122939/http://www.nhsiq. nhs.uk/media/2760650/the_handbook_of_quality_and_service_ improvement_tools_2010.pdf [Accessed 1 June 2021].

26 Institute for Healthcare Improvement. Quality improvement essentials toolkit. IHI, 2017. www.ihi.org/resources/Pages/Tools/QualityImprovement-Essentials-Toolkit.aspx [Accessed 1 June 2021].

27 Curran GM, Bauer M, Mittman B, Pyne JM, Stetler C. Effectivenessimplementation hybrid designs: combining elements of clinical effectiveness and implementation research to enhance public health impact. Med Care 2012;50:217-26.

28 Ommundsen N, Wyller TB, Nesbakken A et al. Preoperative geriatric assessment and tailored interventions in frail older patients with colorectal cancer: a randomized controlled trial. Colorectal Dis 2018:20:16-25.

29 Kocman D, Regen E, Phelps K et al. Can comprehensive geriatric assessment be delivered without the need for geriatricians? A formative evaluation in two perioperative surgical settings. Age Ageing 2019:48:644-49.

Address for correspondence: Ruth de Las Casas, Perioperative Medicine for Older People Undergoing Surgery (POPS), Guy's and St Thomas' NHS Foundation Trust, London, UK. Email: r.delascasas@nhs.net 